\title{
Nachbarschaft ist die Lebendigkeit des Sozialraums
}

\author{
So viel Nachbarschaft wie möglich, \\ so viel professionelle Hilfe wie nötig
}

Klaus Dörner

Prof. Dr. med. Dr. phil. Klaus Dörner

gilt als führender Vertreter der deutschen Sozialpsychiatrie. Er hat Medizin, Soziologie und Geschichte studiert. Von 1980 bis 1996 war er ärztlicher Leiter der Westfälischen Klinik für Psychiatrie, Psychosomatik und Neurologie in Gütersloh. Seit seiner Pensionierung ist er als Referent und Autor tätig. Seit 2003 ist er Mitglied im Präsidium des Deutschen Evangelischen Kirchentages.
Der Raum, in dem jeder Mensch leben und sterben kann, wo er hingehört, kann als »dritter Sozialraum " aufgefasst werden - neben dem privaten und öffentlichen Bereich. Dieser dritte Sozialraum wird angesichts der demografischen Entwicklung an Bedeutung gewinnen und muss im Wohlfahrtsmix seinen eigenständigen Platz erhalten.

In den meisten Dörfern und in fast allen Kleinstädten und Großstadtvierteln entstehen seit etwa drei Jahrzehnten Bürgerinitiativen, die sich um die Integration der wachsenden Zahl pflegebedürftiger und dementer alter Menschen in immer neuen Formen kümmern.

Diese Bewegung strahlt inzwischen auch auf andere hilfe- und integrationsbedürftige Gruppen aus. Diese Bürgerhilfebewegung kann - zusammengefasst als ein seit eineinhalb Jahrhunderten neuartiges Phänomen aufgefasst werden. Bei meiner Feldforschung auf etwa eineinhalb Tausend Reisen an diese bürgerschaftliche Basis in den letzten zehn Jahren war ich bisher wegen historischer Hypotheken skeptisch geblieben, diese neue Bürgerhilfebewegung als Nachbarschaftsbewegung zu bezeichnen.

Inzwischen registriere ich jedoch, dass diese Bürger selbst ihr soziales Tun immer häufiger als » nachbarschaftlich « bezeichnen und es finden sich in der Tat genügend Argumente, die Behauptung aus der Überschrift dieses Artikels zu rechtfertigen.

\section{These: Menschen brauchen Nachbarschaft zum Überleben}

Nach unseren bisherigen Wissen brauchten alle Kulturen der Menschheitsgeschichte die Institution Nachbarschaft sie hätten sonst nicht überleben können. »Nachbarschaft « kann dabei definiert werden als das Engagement der Bürger für fremde Andere in den Grenzen des eigenen Sozialraums. Einzige Ausnahme dieser historisch belegten Erscheinung dürfte die nun zu Ende gehende Epoche der Industriegesellschaft sein.

\section{These: Nachbarn sind Menschen, die mir nahe sind}

Nachbarn sind etymologisch diejenigen Menschen, die in meiner Nähe leben, bauen, wohnen. Denen fühle ich mich zwar nicht wie meiner Familie verpflichtet, jedoch mehr als den Anderen, jenseits der Grenzen meines Sozialraums, den »Fernbauern«. Man kann also hier von einer mittelgradigen Verantwortung sprechen.

\section{These: Der dritte Sozialraum sichert in einer Marktwirtschaft das Gemeinwohl}

Diesem mittleren Verantwortungsgrad entspricht ein mittelgroßes Territorium, nämlich der »dritte Sozialraum « zwischen den Sozialräumen des Privaten und des Öffentlichen. Im städtischen Bereich kann der dritte Sozialraum - je nach Nachbarschaftstradition - fünf bis 3.000 Einwohner umfassend, im ländlichen Bereich 1.000 bis 5.000 Einwohner, jedenfalls nach der international üblichen Konvention. Nur innerhalb dieser räumlichen Grenzen bin ich Nachbar für Nachbarn, was im Übrigen historisch auch für das Territorium einer Kirchengemeinde gilt. Insofern ist dieser dritte Sozialraum der Einzige, der normativ für das Gemeinwohl reserviert ist, da die Sozialräume des Privaten und des Öffentlichen - heute mehr als früher - die Orientierung an den Eigeninteressen erlauben, zumal bei marktwirtschaftlicher Organisation einer Gesellschaft. Ein solches, offenbar menschheitsgeschichtlich bewährtes 
Gleichgewicht zwischen Markt und Gemeinwohl würde brüchig, wenn auch der Sozialraum für das Gemeinwohl als ganzer den Gesetzmäßigkeiten von Angebot und Nachfrage unterfallen würde, wie das heute bei vielen Organisationen des professionellen Helfens der Fall ist.

\section{These: Dem dritten Sozial- raum entspricht eine dritte Sozialzeit}

Analog zum dritten Sozialraum gibt es offensichtlich auch die dritte soziale Zeit der Bürger, zwischen der arbeitsgebundenen und der freien Zeit. Zumindest stößt man darauf, wenn man die engagierten Bürger nach ihren Motiven befragt, wie ich das regelmäßig bei meinen Vortragsreisen tue. Die häufigste Antwort lautet bisher Ausgegrenzten nur im öffentlichen Raum, sind sie nicht integriert; aber bis in meinen Privatraum hinein will ich die Integrationskandidaten auch nicht kommen lassen. Nur im Sozialraum der mittleren Distanz und des mittelgroßen Engagements als Nachbarschaftsraum können diese Menschen auf Toleranz hoffen. Die meisten Menschen sind keine Sozialromantiker: Kaum jemand engagiert sich von Herzen gern nachbarschaftlich, eher schon aus Einsicht in die Notwendigkeit der Hilfe für den Einzelnen und für den Zusammenhalt der Gesellschaft. Das entspricht der empirisch gesicherten Erkenntnis, dass nicht Profis, sondern nur Bürger andere Bürger im Alltag dauerhaft integrieren können. Insofern man sich wenn auch ungern - dazu bekennt, dass irgendwie doch Gemeinwohl und Nachbarschaft das Herzstück des Gemeinwe-

\section{"Jeder Mensch braucht seine Tagesdosis an Bedeutung für Andere"}

nämlich, dass man ein Minimum an sozialem Engagement, "seine Tagesdosis an Bedeutung für Andere « brauche - schon um die übrige freie Zeit von Herzen genießen zu können und um sich - weil ausgelastet - hinreichend gesund fühlen $\mathrm{zu}$ können.

\section{These: Nur Bürger können Bürger integrieren}

Fragt man Schüler, die oft nur den privaten und den öffentlichen Raum kennen, danach, wofür denn wohl ein dritter Sozialraum notwendig sei, dann ist es immer wieder verblüffend, wie wenig Zeit sie brauchen, um die drei menschheitsgeschichtlich wichtigsten Funktionen dieses dritten Sozialraums aufzuzählen: zum Ersten für überlastete Familien, damit sie tragfähig bleiben; zum Zweiten für Alleinstehende, die keine Familie haben; und zum Dritten für alle Prozesse der Integration - nicht nur von hilfsbedürftigen Menschen, sondern beispielsweise auch von Migranten. Insofern ist der dritte Sozialraum als der Wir-Raum die einzige Integrations-Plattform; denn lasse ich die sens sind, wird man sich nicht wundern, dass der dritte Sozialraum als Nachbarschaftsraum in die Räume des Privaten und des Öffentlichen ausstrahlt.

\section{These: Nachbarschaft war schon immer Ausfallbürge für Familie und Sozialstaat}

Die Historiker haben uns belehrt, dass das heutige Reden von der »Großfamilie « ein nostalgischer Wunschtraum ist. Diese gab es höchstens in den Oberschichten, während in der Mittel-und Unterschicht die Familie immer schon ähnlich klein und ähnlich brüchig war wie heute. Deshalb war Nachbarschaft immer schon eine Art Ausfallbürgschaft für die Tragfähigkeit der Normalfamilie und man kann sogar sagen, dass die kleinste tragfähige Einheit immer schon »Familie plus Nachbarschaft « war. Die Blutsverwandten waren in der Vergangenheit und sind auch heute in der Gegenwart sozusagen die Angehörigen ersten Grades und die Nachbarn als Wahlverwandte die Angehörigen zweiten Grades. Insofern ist es bemerkenswert, dass innerhalb weniger
Jahre Nachbarn als »Hauptpflegepersonen « insbesondere in Single-Haushalten, sich von vier auf acht Prozent verdoppelt haben.

\section{These: Nachbarschaft ist ein Element demokratischer Selbstverwaltung}

Was die Ausstrahlung in den öffentlichen Raum angeht, sei daran erinnert: Nachbarschaft gehörte und gehört als von unten ausgehende Selbstorganisation von Bürgern zu den Kernelementen demokratischer Selbstverwaltung. Auch heute steuert lebendige Nachbarschaft den Kitt für die Gesamtgesellschaft bei.

\section{These: Nachbarschaft sichert emotionale Geborgenheit}

Bedeutsam ist der empirische Befund der Heidelberger Gerontologen, wonach bei Befragungen die emotionale Bedeutung an Sicherheit, Geborgenheit und Vertrautheit der Nachbarschaft bei den Bürgern fast genauso groß ist wie die der $\mathrm{Fa}$ milie.

\section{These: Personenorientierte Hilfen brauchen die Nachbarschaft}

Wenig beachtet sind bisher die psychosozialen Folgen des Wandels von der Industrie- zur Dienstleistungsgesellschaft. So lässt sich etwa ein Wandel von der strategisch-großflächigen und fabrikanalogen Organisation des Helfens zur kleinteiligsozialräumlichen und damit nachbarschaftlichen Organisation des Helfens registrieren. Denn in der heutigen Dienstleistungsgesellschaft werden die Menschen nicht mehr zur Hilfe, sondern die Hilfe zu den Menschen gebracht, wenn wir auch erst am Anfang einer solchen Entwicklung stehen. Diese Tendenz wird durch die »Altenexplosion « (und » Kinderimplosion «) beschleunigt, durch die wir in eine Gesellschaft mit dem größten Hilfebedarf der Menschheitsgeschichte (im Gegensatz zum Versprechen der Industriegesellschaft auf Leidensfreiheit) hineinwachsen, was die Rückverlagerung von immer mehr Dienstleistungen von den Profis auf die Bürger als Nachbarn 
erzwingt, um die Profi-Hilfen, wo sie unersetzbar sind, dauerhaft finanzierbar zu halten. Schon entwickelt sich für die Dienstleistungsgesellschaft ein neues Steuerungsprinzip des Helfens: der Bürger-Profi-Mix, was praktisch bedeutet: So viel Nachbarn wie möglich, so viel Profis wie nötig.

\section{These: Inklusion braucht Nachbarschaft}

Als weiterer Beschleuniger der Entwicklung zur Normalisierung von mehr Nachbarschaftsengagement könnte sich die UN-Behindertenrechtskonvention mit ihren Inklusions-Menschenbild erweisen. Denn dessen angelsächsisch-skandinavische Denktradition setzte gegenüber der mehr staatlichen Organisation des Helfens in Deutschland immer schon mehr auf die Eigenschaft der Bürger, sich als Nachbarn für fremde Andere zu engagieren. Mit der Inklusion, so weit ich sie bisher verstehe, geht es nämlich nicht mehr so sehr - wie mit der deutschen Integration - um die isolierte Besonderung einzelner hilfsbedürftiger Individuen oder Gruppen. Ziel von Inklusion ist vielmehr das Miteinander aller Menschen mit und ohne Hilfebedarf, bezogen auf einen bestimmten Sozialraum. Normativ geht es zwar auch um das kostbare Gut von Selbstbestimmung, aber zumindest ebenso sehr um das kostbare Gut der Zugehörigkeit zu einem Sozialraum, zu einer Nachbarschaft, wo alle Menschen mit und ohne Hilfebedarf sich gegenseitig Nachbarn sind.

\section{Resümee}

Wir sind auf dem Wege zu einem neuen Wohlfahrtsmix zwischen Staat, Markt, Zivilgesellschaft und Sozialraum, eine Entwicklung, die auch für die professionelle Soziale Arbeit weitreichende Folgen haben wird.

Während die Stärke der Profis darin besteht, sich spezialisiert auf Zielgruppen zu konzentrieren, was immer auch einen Aussonderungseffekt haben kann, haben Bürger als Nachbarn primär keine "Zielgruppe«. Vielmehr sind sie am Ausgleich zwischen Nehmen und Geben aller Menschen, mit und ohne Hilfebedarf, engagiert und damit an der »Verzwischenmenschli- chung « des jeweiligen Sozialraums als einer radikal gleichberechtigten und daher inklusiven Vielfaltsgemeinschaft.

Zwar können wir diesbezüglich der normativen Aufwertung der Zugehörigkeit gut auf unseren Begriff der Teilhabe aufbauen, haben aber gleichwohl noch zu lernen, dass der jeweilige Sozialraum erst dann vollständig inklusiv ist, wenn alle Menschen, die dort leben oder leben wollen, ihm zugehören, Nachbarn für Nachbarn sind: Inklusion ist ein Sozialraumkonzept!
Da die UN-Konvention auch in Deutschland geltendes Recht ist, haben wir nun alle Chancen, unser aller Nachbarschafts-Seite wieder zu beleben und sie zur Lebendigkeit unseres jeweiligen Sozialraums zu machen. Es wäre für die Jugend schon hilfreich, wenn die sozialen Realitäten der Nachbarschaft, des »dritten Sozialraums « und der »Inklusion « in den Schul-Curricula nicht länger ausgeblendet würden, sondern dort Eingang fänden.

\section{Rückbesinnung auf Werte}

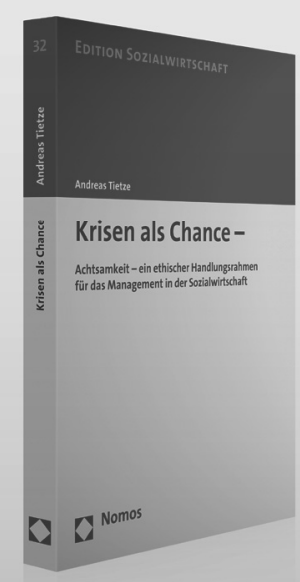

Weitere Informationen: www.nomos-shop.de/13595

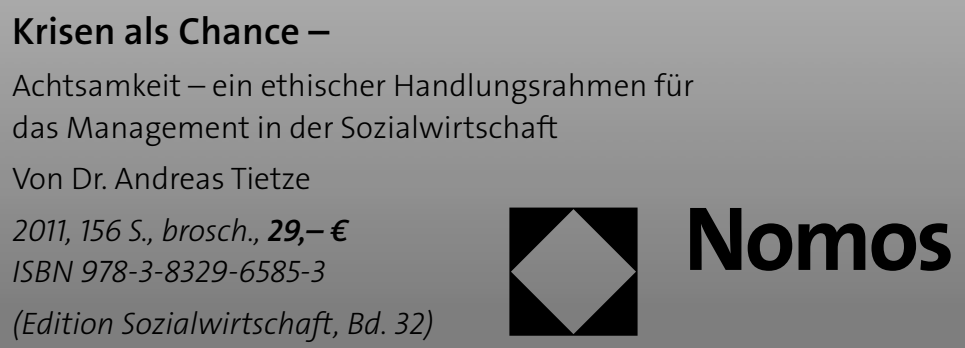

